\title{
ARTICLE
}

\section{EPR Spectra of Alanine Pellets and Lithium Formate Powder in Nuclear Power Plants}

\author{
Hoon CHOI ${ }^{*}$, Byungil LEE, Inbok SUNG, Youngki LIM \\ Radiation Health Research Institute, 388-1, Ssang moon Dong, Do Bong Gu, Seoul, 132-703, Korea
}

\begin{abstract}
Alanine pellets and lithium formate monohydrate powder were installed at the containment buildings of nuclear power plants for environmental radiation monitoring. For some alanine dosimeters, the " $\mathrm{x} / \mathrm{y}$ ratio" of signal was apparently different from that of only gamma exposed dosimeters. Lithium formate powder also showed a difference in appearance and signal saturation. From an analysis of the spectra, both dosimeters appeared to have been exposed to mixed gamma-neutron radiation.
\end{abstract}

KEYWORDS : dosimetry, ESR, EPR, alanine, lithium formate monohydrate, LET

\section{Introduction}

For one or two operating cycles of two nuclear power plants (NPPs), two kinds of dosimeters for electron paramagnetic resonance (EPR) dosimetry, alanine and lithium formate monohydrate, were installed in 30 locations at each nuclear power plant. The purpose was to monitor the radiation level of representative instrumentation and control cables connected to components such as pumps or motors that are important to plant safety. Monitoring was performed by accumulating actual radiation doses for two operating cycles by use of alanine pellets and lithium formate monohydrate powder to assess the aging effects of radiation. EPR or electron spin resonance (ESR) dosimetry is a well known retrospective dosimetry method that can be applied in an emergency after an accident. The dosimetric method detects the free radicals and transforms the energy absorption quantity into amplitude of EPR spectra by use of an EPR spectroscopy system. As a dosimeter for EPR dosimetry, alanine has many useful features including relatively long endurance time of radicals and almost no difference with the radiation dose rate and radiation quality. ${ }^{1,2)}$ Alanine dosimeters have been accepted as transfer dosimeters for their good precision at high radiation range. ${ }^{3,4)}$ For alanine/EPR spectra, it was reported that the peaks of the spectra are due to the three kinds of radicals induced by radiation. ${ }^{5-7)}$ The ratio of the weak "satellite line" and the central peak of the three main alanine spectra (in this study " $\mathrm{x} / \mathrm{y}$ ratio") are changed on the linear energy transfer (LET) value of radiation. ${ }^{8-10)}$ This ratio change can be explained by a decrease of the spinlattice relaxation time $T_{1}$, which is a result of crystallographic defects and plastic deformation in the lattice structure of alanine by high LET radiation such as neutron rays. In spite of the several advantages noted above, the relatively high detection level of alanine dosimeters compared to other dosimeters has researchers to find alternative materials. ${ }^{11)}$ Lithium formate monohydrate was recently identified as a promising dosimetric material. In addition, it was also reported that the peak height in the signal spectra is not easily saturated when lithium formate

*Corresponding Author. Tel.: +82-2-3499-6644; Fax.: +82-2-3499 -6666. E-mail: 93105117@khnp.co.kr

(c) Atomic Energy Society of Japan powder is irradiated with relatively high LET radiation such as neutron rays. ${ }^{12,13)}$ The difference in the peak height ratio was reported to be caused by increased local radical density following the radiation of high LET.

Generally, the radiation that mainly affects the aging process of cables is known to be gamma rays. Therefore, the main purpose of dose measurement in NPPs is the estimation of gamma dose. However, the results of experiment have shown that the "x/y ratio" of several alanine dosimeters returned from NPPs is lower than that of only gamma ray-exposed dosimeters. For lithium formate powder, the difference in the signal ratio according to power variation is not as clear as that of alanine dosimeters. This may be caused by the fact that some lithium formate powder in capsules loses its moisture by incomplete sealing in NPPs. The lost of moisture could account for much lower doses than those anticipated based on the results from alanine dosimeters. This relationship between moisture and signal intensity was experimented and the results were used in the process of selecting proper dosimeters for this study. In this experiment, only five locations in the containment building were selected for comparison of spectrum shape, because most of the locations in NPPs have no chance to be exposed to mixed radiation composed of gamma rays and neutrons. Experiments were conducted using two different kinds of EPR dosimeters that have variations in the shape of spectra or changes in the power saturation of the signal intensity on the radiation quality. It was shown that the features of the two dosimeters could be used to discriminate radiation quality in NPPs.

\section{Experiment}

\section{Dosimeter and Spectrometry System}

Polycrystalline lithium formate monohydrate powder $\left(\mathrm{HCO}_{2} \mathrm{Li} \cdot \mathrm{H}_{2} \mathrm{O}, 98 \%\right)$ from Sigma-Aldrich Co. and pallet type alanine dosimeters from BioMax (Germany) that contained $\alpha$-amino acid alanine, $\mathrm{CH}_{3}-\mathrm{CH}\left(\mathrm{NH}_{2}\right)-\mathrm{COOH}$, and Teflon as a binding material(ratio $>9$ ) were purchased and stored in darkness for the experiments. EPR dosimetry for 
alanine dosimeters was carried out by using "e-scan", which was manufactured for the sole purpose of dosimetry of alanine pellets. "e-scan" was calibrated by standard alanine dosimeters irradiated in the National Physical Laboratory (NPL, United Kingdom) using cobalt-60 at a range of 25 to $5 \mathrm{kGy}$.

The same alanine dosimeters were also used as reference dosimeters for comparison of the spectra shape by an EMX spectrometer (Bruker, Germany). An EMX spectrometer is generally used in routine EPR research. Temperature and humidity meters for monitoring environmental conditions were installed inside the cavity of the EMX spectrometer. Signal dependency on power, signal fading, and annealing of lithium formate powder were measured by the dosimeters which were exposed to cesium-137 at a range of 50 to 3,000 Gy and a dose rate of $6 \mathrm{~Gy} / \mathrm{min}$ using a blood irradiator (IBL 437C), which was installed at the research institute (Radiation Health Research Institute).

\section{Installation and Measurement of Dosimeter}

To prevent accidental shock, plastic capsules that contained the dosimeters were carefully fastened to the power and instrument cables. After one or two operating cycles, the installed capsules were returned from the NPPs and all the dosimeters were stored under controlled temperature and humidity conditions (temperature: 21 $\sim 26^{\circ} \mathrm{C}$, relative humidity: $16 \sim 22 \%$ ). A total of 5 locations out of 60 locations in NPPs were selected for their relatively high dose levels and were compared in terms of "x/y ratio" and saturation behavior. These locations were near the primary loop lines or outside of the first barrier around the reactors in the containment building. The absorbed dose of the alanine dosimeters was measured by "e-scan" and corrected by parameters such as the weight of each pellet and the average temperature at each location, which was measured by temperature monitors in the NPPs. When the "x/y ratio", that is, the No $7(\mathrm{x})$ over No $8(\mathrm{y})$ peak ratio, shown in Fig. 1, was measured, the environmental factors were more severely controlled than were the dosimeter storage conditions (temperature: 23.4 24. $1^{\circ} \mathrm{C}$, relative humidity: $17 \sim 20 \%$ ).

In particular, the " $\mathrm{x} / \mathrm{y}$ ratio" of the alanine dosimeters was highly sensitive to variation of temperature and humidity. All the data were measured on a daily basis for three weeks and the data measured in the outer range of the temperature and humidity limits were discarded for precise measurements. The " $\mathrm{x} / \mathrm{y}$ ratio" was very sensitive to factors such as angle and position of the dosimeter in the cavity, Q value movement, and the time for attaining equilibrium in the cavity. Uncertainties involved in measurement of the dosimeters are shown in Table 1. The spectroscopy system of alanine dosimeters and lithium formate powder used for comparison of the spectra was EMX spectrometer equipped with X-band bridge. Signal intensity change of lithium formate powder depending on the decrease of mass by the heating process was also measured by an EMX spectrometer with a manganese standard sample for reference in a cavity. Microwave power and field modulation were $0.2 \mathrm{~mW}$ and $50 \mathrm{~mW}$ and $1 \mathrm{mT}$ for lithium formate powder. In the case of alanine dosimeters, microwave power was $30 \mathrm{~mW}$ and field modulation was $0.2 \mathrm{mT}$ for the measurement.

Table 1 Uncertainties of measured dose and "x/y ratio"

\begin{tabular}{|c|c|c|c|c|}
\hline & Dose & $250 \mathrm{~Gy}$ & $1,000 \mathrm{~Gy}$ & $3,000 \mathrm{~Gy}$ \\
\hline \multirow{3}{*}{$\begin{array}{c}\text { Alanine } \\
\text { pellets }\end{array}$} & $\begin{array}{l}\text { "e-scan" } \\
\text { (expanded combined } \\
\text { uncertainty) }\end{array}$ & $5.33 \%$ & $2.97 \%$ & $2.44 \%$ \\
\hline & $\begin{array}{l}\text { EMX spectroscopy } \\
\text { response variation } \\
\text { of " } \mathrm{x} / \mathrm{y} \text { ratio" }\end{array}$ & $0.91 \%$ & $0.25 \%$ & $0.16 \%$ \\
\hline & $\begin{array}{l}\text { EMX spectroscopy } \\
\text { repetition variation } \\
\text { of " } \mathrm{x} / \mathrm{y} \text { ratio" }\end{array}$ & $0.80 \%$ & $0.34 \%$ & $0.12 \%$ \\
\hline \multirow{2}{*}{$\begin{array}{l}\text { Lithium } \\
\text { formate } \\
\text { powder }\end{array}$} & $\begin{array}{l}\text { EMX spectroscopy } \\
\text { response variation } \\
\text { of " } \mathrm{x} / \mathrm{y} \text { ratio" }\end{array}$ & & $1.93 \%$ & \\
\hline & $\begin{array}{l}\text { EMX spectroscopy } \\
\text { repetition variation } \\
\text { of " } \mathrm{x} / \mathrm{y} \text { ratio" }\end{array}$ & & $0.54 \%$ & \\
\hline
\end{tabular}

\section{Results and Discussion}

The typical spectrum of alanine pellets and lithium formate powder that was irradiated with gamma rays is displayed in Fig. 1. The vertical lines in the spectrum of alanine show readout positions of the $\mathrm{x}$ and $\mathrm{y}$ lines. The spectrum of lithium formate powder consists of a single resonance line having broad line width.

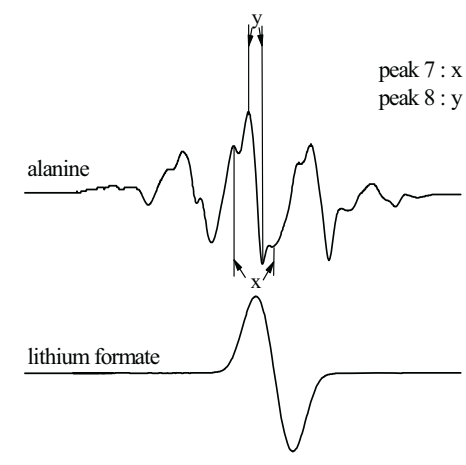

Fig. 1 Typical EPR spectra of alanine dosimeters and lithium formate powder irradiated with cobalt-60 at $500 \mathrm{~Gy}$. The satellite peak and main resonance peak of alanine are indicated as " $x$ " and " $y$ " letters in the figure and used in the calculation of the " $\mathrm{x} / \mathrm{y}$ ratio".

As in Table 2, the " $\mathrm{x} / \mathrm{y}$ ratio" of five alanine dosimeters from the containment building of NPP No.1 ("Yonggwang", pressurized water reactor type) and NPP No.2 ("Ulchin", pressurized water reactor type) was measured. The ratio of two locations (No 15, No 27) from NPP No.1 and one location (No 18) from NPP No.2 was much lower than that of gamma-exposed dosimeters in NPL. The remaining alanine dosimeters (No 3, No 17) did not show a decrease in the "x/y ratio". The measured value of the "x/y ratio" in the alanine dosimeters was unstable and frequently changed by the environmental and measurement conditions. Thus, 
the measured " $\mathrm{x} / \mathrm{y}$ ratio" of the alanine dosimeters from NPPs were corrected for environmental factors and normalized to the ratio of gamma exposed alanine dosimeters, as outlined in the below Fig. 2. The alanine pellet at the location of No 27 appears to have been exposed to mixed radiation with higher effective LET than the alanine pellet at location No 18. In the case of lithium formate powder, after being irradiated with gamma rays of 1,000 Gy, the signal intensity was decreased, as shown in Fig. 3. About 70 hours after irradiation, the signal intensity stabilized. Therefore, all the lithium formate powder was stored for at least three days after irradiation in this experiment.

Table 2 Estimated dose and "x/y ratio" of alanine dosimeters.

\begin{tabular}{lccccc}
\hline \multicolumn{1}{c}{ No } & $\begin{array}{c}\text { No 15 } \\
\text { (NPP No.1) }\end{array}$ & $\begin{array}{c}\text { No 27 } \\
\text { (NPP No.1) }\end{array}$ & $\begin{array}{c}\text { No 3 } \\
\text { (NPP No.2) }\end{array}$ & $\begin{array}{c}\text { No 17 } \\
\text { (NPP No.2) }\end{array}$ & $\begin{array}{c}\text { No 18 } \\
\text { (NPP No.2) }\end{array}$ \\
\hline \hline $\begin{array}{l}\text { Absorbed } \\
\text { dose (e-scan) }\end{array}$ & 540 Gy & 1,993 Gy & 347 Gy & 254 Gy & 2,133 Gy \\
\hline $\begin{array}{l}\text { "x/y ratio" } \\
\text { (normalized) }\end{array}$ & 0.7594 & 0.7147 & 0.7704 & 0.7634 & 0.7318 \\
\hline
\end{tabular}

* Only gamma ray exposed alanine dosimeter ratio is about $0.7700(1.0000)$

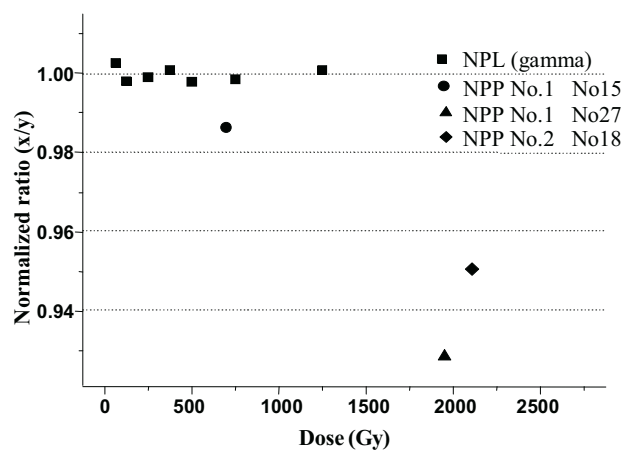

Fig. 2 The "x/y ratio" of alanine pellets that were irradiated at NPL to gamma dose and returned from NPPs after one or two fuel cycles.

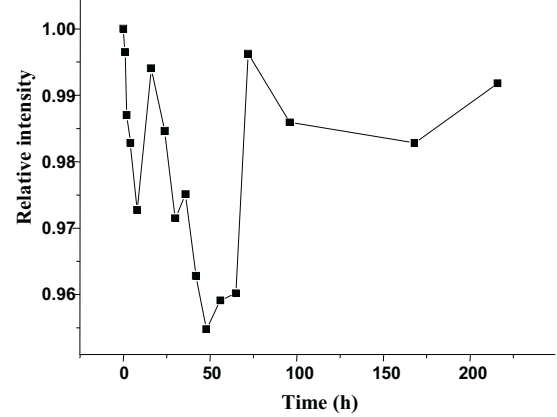

Fig. 3 The fading of EPR spectra of lithium formate powder after irradiation of gamma rays for the first 200 hours.

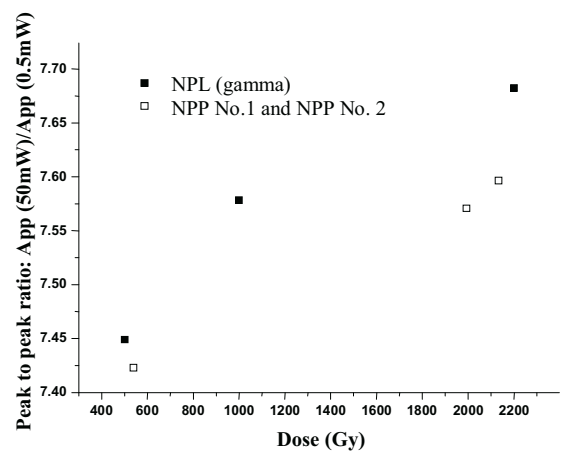

Fig. 4 The ratio of the signal amplitude obtained at two different microwave powers $(0.5$ and $50 \mathrm{~mW})$ for lithium formate dosimeters irradiated with only gamma rays (black) and mixed radiation from a nuclear power plant (white). Lithium formate powders are No 15 and No 27 from NPP No.1 and No 18 from NPP No.2.

The ratio of signal intensity at two different powers was evaluated on a daily basis. This difference in saturation characteristics between the two groups of lithium formate powders can be observed in Fig. 4. In the plot, the power level is changed from $0.5 \mathrm{~mW}$ to $50 \mathrm{~mW}$ and the signal intensity ratio changed according to radiation quality. The measured difference was very subtle and in most cases it could be accounted for by the movement of the quartz tube inside the cavity. Three lithium formate powders (No 15, 27, 18) showed slightly low ratios. The remaining samples did not show apparent differences. These experimental results are consistent with the results of alanine dosimeters at the locations in the containment buildings.

Some lithium formate powders from NPPs lost their original transparency and became white in appearance. This seems to be caused by moisture evaporation by incomplete sealing and relatively high temperature at the installed locations in the NPPs. The evaporation process was reproduced by heating the powder in an unsealed capsule. Lithium formate powder that had already been exposed to gamma rays of 1,800 Gy was prepared and heated for 150 minutes in opened and unopened capsules. The signal intensity of the powder in the opened capsule decreased rapidly by heating, as shown in Fig. 5. In the experiment, there was no change in the signal intensity or appearance in the case of completely sealed capsules. It appears that the dehumidification process of lithium formate powder in the capsules induced the change in the appearance and the destruction of radicals produced in a lattice structure of the lithium formate powder. Based on the results of the above experiments, lithium formate powders with changed appearance and very low signal intensity were subsequently not used for dose estimation. 


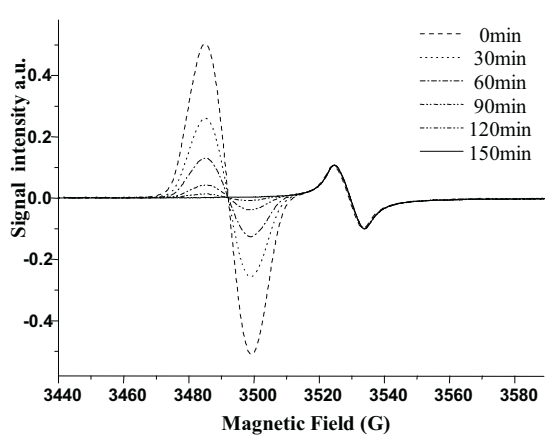

Fig. 5 Decrease of signal amplitude of lithium formate powder, which was annealed by an electric heater at a temperature of 343 $\mathrm{K}$ for 150 minutes; after a half hour of heating, the lithium formate powder was scanned by an EMX spectrometer and heated again five times. The right side peak is the standard manganese sample for reference.

\section{Conclusion}

The spectrum shape of some alanine dosimeters and lithium formate powder installed in the containment buildings of NPPs showed differences in comparison with dosimeters exposed only to gamma rays. In the case of alanine dosimeters, there was an apparent change of spectra, expressed as the "x/y ratio". As noted in other papers $9,10,13$, high LET radiation such as neutron rays causes shape changes of the spectrum of alanine dosimeter and lithium formate powder. Thus, the unanticipated high dose level and low "x/y ratio" of some alanine dosimeters from the containment building could be explained by exposure to mixed radiation with high LET. Generally, the locations evaluated in this study are regarded as being exposed only to gamma rays, because the positions are blocked from direct neutron rays from the reactor by thick cement barriers and heavy instruments. The variation of spectra shape at the locations is most likely due to indirect irradiation of neutron rays from the reactor through gaps between the primary loop lines and holes in the barrier.

Although a limited number of samples were experimented, the results show that the spectrum change of EPR dosimeters could be used as an indicator for the discrimination of radiation quality in NPPs.

\section{References}

1) IAEA, Standard Practice for Use of the Alanine-EPR Dosimetry System, ISO/ASTM E1607-94 (1994).

2) S. Olsson and E. S. Bergstrand, "Calibration of alanine dosimeters," ISRN ULi-RAD-R-92-SE, Report 92, Linköping University, Sweden (2001).

3) K. Mehta and R. Girzikowsky, "Reference dosimeter system of the IAEA," Radat. Phys. Chem., 46[46], 1247 (1995).

4) J. M. Dolo, N. lecerf and I. Tartes, "Progress in Quality Control of Alanine Dosimeters" Appl. Radiat. Isot., 47[11-12], 1165 (1996).

5) E. Malinen, M. Z. Heydari, E. Sagstuen and E. O. Hole, "Alanine Radicals, Part 3: Properties of the components contributing to the EPR spectrum of $x$-irradiatied alanine dosimeters," Radiation Research, 159, 23 (2003).

6) M. Z. Heydari, E. Malinen, E. O. Hole and E. Sagstuen, "Alanine Radicals 2. The Composite Polycrystalline Alanine EPR Spectrum Studied by ENDOR, Thermal Annealing, and Spectrum Simulations," J. phys. chem. A, 106[38], 8971 (2002).

7) E. Malinen, E. A. Hult, E. O. Hole and E. Sagstuen, "Alanine Radicals, Part 4: Relative Amounts of Radical Species in Alanine Dosimeters after Exposure to 6-19Mev Electrons and $10 \mathrm{Kv}-15 \mathrm{Mv}$ photons," Radiation Research, 150, 149 (2003).

8) B. Ciesieelski, L. Wielopolski, "The effects of boron on the Electron paramagnetic resonance spectra of alanine irradiated with thermal neutrons," Radiation Research, 144, 59 (1995).

9) B. Ciesielski, L. Wielopolski, "The Effects of Dose and Radiation Quality on the Shape and Power Saturation of the EPR Signal in Alanine," Radiation Research, 140, 105 (1994).

10) B. Ciesielski, Z. Stuglik, L. Wielopolski and I. Zvara, "The Effect of High-Linear Energy Transfer Ions on the Electron Paramagnetic Resonance Signal in Alanine," Radiation Research, 150, 469 (1998).

11) M. Ikeya, G. M. Hassan, H. Sasaoka, Y. Kinoshita, S. Takaki, C. Yamanaka, "Strategy for finding new materials for ESR dosimeters," Applied Radiation and Isotopes, 52[5], 1209 (2000).

12) T. A. Vestad, E. Malinen, A. Lund, E. O. Hole, E. Sagstuen, "EPR dosimetric properties of formates," Applied Radiation and Isotopes, 59, 181 (2003).

13) E. Malinen, E. Waldeland, E. O. Hole, E. Sagstuen, "LET effects following neutron irradiation of lithium formate EPR dosimeters," Spectrochimica Acta Part A: Molecular and Biomolecular Spectroscopy, 63[4], 861 (2006). 